\title{
Correlación y concordancia entre instrumentos de control del asma en niños
}

\author{
ALBERTO VIDAL G.*, ANA MARÍA ESCOBAR C.* y MARÍA EUGENIA MEDINA R.**
}

Correlation and agreement between the instruments of asthma control in children

Introduction: It has been shown that asthma control and quality of life in children have moderate correlation, however FEV ${ }_{1}$ would have only a weak correlation with these control parameters. Our objective was to measure clinical correlation and concordance between parameters of pediatric asthma control in Chilean children. Methods: Study carried out in the Unit of Respiratory Diseases, Roberto del Río Children's Hospital, between November 2009 and May 2010, in a series of persistent asthmatic children between 12 and 17 years old. We measured the correlation and agreement between asthma quality of life (PAQLQ Juniper), asthma control (ACT) and FEV (percentage predicted). We used coefficient of Spearman rank correlation (rs) and kappa index. The sample size was calculated considering a power of $80 \%$ and $p<0.05$. Results: We enrolled 88 patients, average age 13.4 years old, 61\% male. Correlation PAQLQ and ACT (rs: 0.48), ACT and FEV (rs: 0.11), PAQLQ and FEV (rs:-0.1). Concordance PAQLQ $<6$ points and ACT $<20$ points (kappa: 0.46). Concordance ACT $<20$ points with $F E V_{1}<80 \%$ (kappa: -0.02), concordance PAQLQ < 6 points with $F E V_{1}<80 \%$ (kappa: -0.02).Conclusions: The quality of life and asthma control have moderate correlation. FEV $V_{1}$ weakly correlation with ACT and negative correlation with PAQLQ. The best cut-off point to identify uncontrolled asthma was obtained with ACT $<20$ points and PAQLQ Juniper $<6$ points. FEV $1<80 \%$ do not have good agreement with ACT and PAQLQ to detect uncontrolled asthma.

Key words: Asthma control, quality of life, lung function.

\section{Resumen}

Introducción: Se ha demostrado que el control del asma y la calidad de vida en niños se correlacionan en forma moderada, sin embargo, el $V E F_{1}$ sólo tendría una correlación débil con dichos indicadores de control. El objetivo de este estudio fue medir la correlación clínica y concordancia entre indicadores de control del asma pediátrica en niños chilenos. Pacientes y Métodos: Estudio realizado en el policlínico de enfermedades respiratorias del Hospital Roberto del Río, entre noviembre de 2009 y mayo de 2010, con un grupo de asmáticos persistentes de 12 a 17 años. Se midió la correlación y concordancia entre calidad de vida (PAQLQ de Juniper), control del asma (ACT) y VEF 1 (porcentaje del predicho). Se utilizó el coeficiente de correlación por rangos de Spearman (rs) e índice de kappa. El tamaño muestral se calculó considerando un poder de $80 \%$ y p < 0,05. Resultados: Se enrolaron 88 pacientes, promedio de edad 13,4 años, 61\% de género masculino. Correlación PAQLQ y ACT (rs: 0,48), ACT y VEF 1 (rs: 0,11), PAQLQ y VEF $($ (rs:-0,1). Concordancia PAQLQ $<6$ puntos y ACT $<20$ puntos (kappa: 0,46). Concordancia ACT $<20$ puntos con $V E F_{1}<80 \%$ (kappa: -0,02), concordancia $P A Q L Q<6$ puntos con $V E F_{1}<80 \%$ (kappa: -0,02). Conclusiones: La calidad de vida y el control del asma tienen correlación moderada. El VEF $F_{1}$ presenta correlación débil con ACT y negativa con $P A Q L Q$. El mejor punto de corte para detectar asma no controlada se obtuvo con ACT $<20$ puntos y $P A Q L Q$ de Juniper < 6 puntos. EL VEF $1<80 \%$ no tiene buena concordancia con ACT y PAQLQ para detectar asma mal controlada.

Palabras clave: Control de asma, calidad de vida, función pulmonar.

* Pediatra Especialista en Enfermedades Respiratorias Infantiles. Hospital Roberto del Río.

** Tecnólogo Médico Hospital Roberto del Río. 


\section{Introducción}

El control del asma puede ser medido a través de varios métodos. Entre los más importantes se conocen las encuestas de control de síntomas, las encuestas de calidad de vida, la función pulmonar, especialmente el volumen espiratorio forzado en el primer segundo $\left(\mathrm{VEF}_{1}\right)$ y la concentración de óxido nítrico exhalado (FeNO). Las primeras publicaciones que validaron estos instrumentos en adultos asmáticos, notificaron buenos grados de correlación entre calidad de vida y control del asma. En dichos estudios se aplicó el Asthma Quality of life Questionnaire en sus versiones extensa y abreviada (AQLQ o miniAQLQ) y control del asma medido por el Asthma Control Questionnaire (ACQ) o por el Asthma Control Diary $^{1-4}$. Sin embargo, sólo se ha notificado una débil correlación entre control o calidad de vida en asma y $\mathrm{VEF}_{1}$, el cual es uno de los indicadores utilizados por las guías clínicas en la monitorización del control del asma ${ }^{5-8}$. En niños y adolescentes asmáticos se ha encontrado moderada correlación entre el control clínico medido por ACQ, calidad de vida medida por el Paediatric Asthma Quality of Life Questionnaire (PAQLQ) o por el Adolescent Asthma Quality of Life Questionnaire (AAQOL) y síntomas de asma. Estos mismos estudios han demostrado nula o débil correlación entre control del asma o calidad de vida con $\mathrm{VEF}_{1}$ o $\mathrm{PEF}^{9-11}$. Se ha notificado grados moderados de correlación y concordancia entre control medido según recomendación GINA y ACT en adultos y adolescentes asmáticos ${ }^{12}$. Nuestro grupo ha publicado dos investigaciones en el tema. En un estudio se notificó concordancia débil entre control medido por encuesta GINA y ACT en adolescentes con asma persistente. En el otro se demostró la prevalencia de alteración de la calidad de vida medida por PAQLQ en niños asmáticos y sus cuidadores ${ }^{13,14}$. En estos estudios no se incluyó $\mathrm{VEF}_{1}$, ni se estableció relación entre las variables de control del asma pediátrica. El presente estudio tiene dos objetivos: a) establecer el grado de relación entre variables implicadas en el control del asma pediátrica, lo que se conoce como correlación; b) medir el grado de acuerdo o coincidencia que tienen estos indicadores en la evaluación del control, término denominado concordancia.

\section{Pacientes y Métodos}

Entre noviembre de 2009 y mayo de 2010 enrolamos a 88 pacientes asmáticos persistentes entre 12 y 17 años, controlados en la unidad de
Enfermedades Respiratorias del Hospital Roberto del Río. Se realizó una selección no aleatoria de dos pacientes diarios, de entre aquellos pacientes asmáticos citados a espirometría de control en el laboratorio de función pulmonar. La muestra correspondió al $10 \%$ del total de pacientes que realizaron espirometría durante el periodo del estudio. Utilizamos la clasificación GINA ${ }^{6}$ para evaluar la severidad del asma. Se aplicó la encuesta de control de asma ACT para niños mayores de 12 años y adultos en su versión en español con escala de 5 a 25 puntos, definiéndose asma no controlada aquella con puntaje inferior a 20 puntos $^{15-17}$. Se aplicó el Cuestionario de Calidad de Vida PAQLQ versión en español de Juniper con 23 preguntas distribuidas en tres dominios (síntomas, emocional y limitación de actividades) graduada con escala ordinal de Likert de 1 a 7 puntos de promedio ${ }^{9,18}$. Se realizó espirometría a todos los pacientes obteniendo el valor del $\mathrm{VEF}_{1}$ (porcentaje del valor predeterminado). La espirometría fue realizada por un médico especializado en estudio funcional respiratorio, con un espirómetro Jaeger modelo MS-PFT, utilizando los valores predictivos de Knudson y cols. Para establecer el grado de correlación de estas variables, se utilizó el coeficiente de correlación de Spearman. El grado de concordancia se midió mediante índice de kappa. El tamaño muestral necesario para el estudio fue considerado con un poder de $80 \%$ con un valor de $\mathrm{p}<0,05$.

\section{Resultados}

La muestra estudiada correspondió a 88 pacientes asmáticos entre 12 y 17 años, con un promedio de edad de 13,4 años. El 61\% correspondió a varones. Según la clasificación de severidad de GINA encontramos: persistentes leves en $25 \%$, persistentes moderados en $51,1 \%$ y persistentes severos en $23,9 \%$. El puntaje de control ACT promedio fue 19,5 puntos (rango de 6 a 25). El 44,3\% no tenía control de la enfermedad según ACT (puntaje < 20) y el 12,5\% fue clasificado como asma pobremente controlada (puntaje $<15$ ). Los puntajes promedio de calidad de vida se distribuyeron de la siguiente forma: calidad de vida general 5,4 puntos (rango de 2 a 7), calidad de vida en dominio síntomas 5,3 puntos (rango de 1,9 a 7), calidad de vida en dominio emocional 5,5 puntos (rango de 1,6 a 7) y calidad de vida en dominio limitación de actividades 5,4 puntos (rango de 2,4 a 7). El 82\% de los pacientes con asma no controlada por ACT tenía promedio de calidad de vida de Juniper inferior a 6 puntos. El 
$65 \%$ de los pacientes con promedio de calidad de vida de Juniper inferior a 6 puntos se clasificó como asma no controlada según ACT. El promedio de $\mathrm{VEF}_{1}$ fue 106,2\% (rango: $51,9-154 \%)$. Sólo un paciente $(1,1 \%)$ tenía $\mathrm{VEF}_{1}<80 \%, 74$ $(84,1 \%)$ tenían $\mathrm{VEF}_{1}$ entre 80 y $120 \%$ y $13(14,8 \%)$ sobre $120 \%$. El $45 \%$ de los pacientes con $\mathrm{VEF}_{1}$ igual o superior a $80 \%$ fue clasificado como asma sin control según ACT y el $56 \%$ tenía calidad de vida de Juniper inferior a 6 puntos. En la Tabla 1 se muestran los coeficientes de correlación por rangos de Spearman entre el control del asma, la calidad de vida y $\mathrm{VEF}_{1}$. En la Tabla 2 se muestra el grado de concordancia obtenido por índice de kappa entre el control de asma, diferentes promedios de calidad de vida y $\mathrm{VEF}_{1}$.

Tabla 1 Correlación de Spearman (rs) entre control del asma, calidad de vida y $\mathrm{VEF}_{1}$ en 88 pacientes entre 12 y 17 años de edad

\begin{tabular}{|clllll|}
\hline & CVG & CVS & CVE & CVLA & VEF $_{1}$ \\
ACT & $0,48^{* *}$ & $0,55^{* *}$ & $0,42^{* *}$ & $0,35^{* *}$ & $0,11^{*}$ \\
$\mathrm{VEF}_{1}$ & $-0,1$ & $-0,05$ & $-0,07$ & $-0,08$ & \\
\hline
\end{tabular}

ACT: Asthma Control Test. CVG: calidad de vida general. CVS: calidad de vida dominio síntomas. CVE: calidad de vida dominio emocional. CVLA: calidad de vida dominio limitación de actividades. VEF 1 : volumen espiratorio forzado en el primer segundo. ${ }^{*} \mathrm{p}<0,05 ;{ }^{*} \mathrm{p}<0,001$.

Tabla 2 Concordancia medida por índice de kappa entre control del asma, $\mathrm{VEF}_{1} \mathrm{y}$ calidad de vida general en 88 pacientes entre 12 y 17 años de edad

\begin{tabular}{|c|c|c|c|c|c|c|}
\hline & $\begin{array}{c}\text { ACT } \\
<20 \\
\mathbf{n}\end{array}$ & $\begin{array}{c}\text { ACT } \\
\geq 20 \\
\mathbf{n}\end{array}$ & kappa & $\begin{array}{c}\mathrm{VEF}_{1} \\
<\mathbf{8 0 \%} \\
\mathbf{n}\end{array}$ & $\begin{array}{c}\mathrm{VEF}_{1} \\
>\mathbf{8 0} \% \\
\mathrm{n}\end{array}$ & kappa \\
\hline $\mathrm{CVG}<3$ & 2 & 0 & & 0 & 2 & \\
\hline $\mathrm{CVG}>3$ & 37 & 49 & $0,05^{*}$ & 1 & 85 & $-0,01$ \\
\hline $\mathrm{CVG}<4$ & 7 & 4 & & 0 & 12 & \\
\hline $\mathrm{CVG}>4$ & 32 & 45 & $0,1^{*}$ & 1 & 75 & $-0,02$ \\
\hline $\mathrm{CVG}<5$ & 24 & 9 & & 0 & 33 & \\
\hline $\mathrm{CVG}>5$ & 15 & 40 & $0,44 * *$ & 1 & 54 & $-0,02$ \\
\hline $\mathrm{CVG}<6$ & 32 & 17 & & 0 & 49 & \\
\hline $\mathrm{CVG}>6$ & 7 & 32 & $0,46^{* *}$ & 1 & 38 & $-0,02$ \\
\hline $\mathrm{CVG}<7$ & 39 & 46 & & 1 & 84 & \\
\hline $\mathrm{CVG}>7$ & 0 & 3 & $0,05^{*}$ & 0 & 3 & 0,0008 \\
\hline $\mathrm{ACT}<20$ & & & & 0 & 48 & \\
\hline $\mathrm{ACT} \geq 20$ & & & & 1 & 39 & $-0,02$ \\
\hline
\end{tabular}

ACT: Asthma Control Test. CVG: calidad de vida general de Juniper, $\mathrm{VEF}_{1}$ : volumen espiratorio forzado en el primer segundo, ${ }^{*} \mathrm{p}<0,05$; $* * \mathrm{p}<0,01$.

\section{Comentarios}

Los adultos y niños asmáticos que no alcanzan el control de la enfermedad, desarrollan alteraciones en diferentes áreas de su calidad de vida. En asma pediátrica, la falta de control también se asocia a problemas emocionales, ausentismo laboral y deterioro la calidad de vida del cuidador ${ }^{14,19,20}$. Encontramos una correlación moderada entre control de asma y calidad de vida, coincidiendo con los estudios citados previamente. Al analizar los tres tipos de dominios de la calidad de vida general, destacamos que la mejor correlación con ACT se logró con el dominio de síntomas de asma, luego el domino de la función emocional y finalmente con el dominio de limitación en las actividades de la vida cotidiana, lo que corrobora lo publicado por Schatz et al, quienes demostraron que las encuestas de control reflejan bien los dominios de síntomas o actividad en la calidad de vida, pero bastante menos los dominios emocionales o ambientales ${ }^{21}$. Ambos instrumentos (ACT y PAQLQ) poseen preguntas bastante similares en el ámbito de la evaluación de los síntomas, situación que con mucha frecuencia es la única en ser considerada por el clínico. Sin embargo, los cuestionarios de calidad de vida miden áreas no evaluadas por las encuestas de control que son igualmente importantes, como los sentimientos y las percepciones emocionales subjetivas, especialmente en los adolescentes, mediante las cuales es posible pesquisar trastornos psiquiátricos, susceptibles de ser evaluados por especialistas en salud mental ${ }^{22,23}$. Los estudios de validación inicial del PAQLQ de Juniper no establecen puntajes de corte para definir mala calidad de vida o asma no controlada, ya que están orientados al control del tratamiento, por medio de cambios en el promedio personal de calidad de vida, el cual puede ser medido por un coeficiente de variación ${ }^{8,9}$. Sin embargo, en el presente estudio se encontró una concordancia moderada entre ACT inferior a 20 puntos y calidad de vida 
general menor a 6 puntos. Un estudio reciente que utilizó estas mismas encuestas, mostró resultados muy similares, demostrando que ambos instrumentos son capaces de identificar en niños el asma no controlada de la controlada. En dicho estudio se estableció que el valor de $\mathrm{ACT}<20$ puntos o el puntaje de PAQLQ de Juniper $<6,2$ puntos son los mejores puntos de corte para detectar la falta de control del asma pediátrica ${ }^{24}$.

Se encontró una correlación débil y concordancia negativa entre ACT y $V_{E F}$. Similares resultados se han reportado en niños y adolescentes, en los que las encuestas de control de síntomas se han encontrado superiores a la medición de $\mathrm{VEF}_{1}, \mathrm{FeNO}$ y $\mathrm{PEF}$, en la toma de decisiones y cambio del tratamiento del asma ${ }^{25,26}$.

$\mathrm{ACT}$ es un instrumento que realiza una medición subjetiva, pero muy útil en el control ambulatorio de la mayoría de los niños asmáticos, independiente de su severidad. El $\mathrm{VEF}_{1}$, aunque entrega una evaluación objetiva, tiene valores normales en la mayoría de los niños con asma. Bacharier et al, publicaron un estudio en niños con asma persistente moderada, en el cual notificaron cerca de un $94 \%$ de pacientes con valores de $\mathrm{VEF}_{1}$ superior a $80 \%$ del valor predeterminado, sin correlación con los grados de severidad propuestos por las guías de control ${ }^{27}$. En nuestro estudio el $98 \%$ de los pacientes, que en su mayoría eran asmáticos persistentes leves a moderados, tenían $\mathrm{VEF}_{1}$ superior a $80 \%$ del predeterminado. Por esta razón y a diferencia del los adultos con asma, el $\mathrm{VEF}_{1}$ no sería un instrumento adecuado para monitorizar el control del asma pediátrica. La excepción a lo anterior son los asmáticos severos o los catalogados como asmáticos de difícil control, en los que está claro el valor que el $\mathrm{VEF}_{1}$ tiene en el diagnóstico y el seguimiento ${ }^{28,29}$.

Para mejorar el rendimiento del $\mathrm{VEF}_{1}$ en el control del asma pediátrica e identificar con mayor exactitud a los asmáticos sin control adecuado, un grupo de autores recomiendan elevar el punto de corte en el valor porcentual del $\mathrm{VEF}_{1}$ de $\geq 80 \%$ a $\geq 100 \%$, sin embargo, estos resultados deben ser replicados por más estudios antes de ser considerados por las guías de manejo en asma ${ }^{30}$.

Otros proponen utilizar valores de la espirometría que podrían tener mejor correlación con la severidad del asma, especialmente en asma persistente leve a moderada y que podrían ser de mayor utilidad en el seguimiento, como la relación $\mathrm{VEF}_{1} / \mathrm{CVF}$ o el porcentaje de cambio del $V_{E F}$ en respuesta a los agonistas adrenérgicos $\beta-2^{31}$. Pensamos que sería muy relevante complementar este estudio con otros diseños que evalúen correlación o concordancia entre estos parámetros y el control del asma en el niño.

La calidad de vida general y con diferentes promedios como puntos de corte se correlacionó y concordó negativamente con el valor del $\mathrm{VEF}_{1}$. Resultados similares han sido comunicados en publicaciones previas en niños asmáticos ${ }^{32,33}$.

Debemos mencionar dos limitaciones del estudio. La primera fue la falta de medición de inflamación bronquial mediante FeNO, examen recomendado por los consensos internacionales en la evaluación del control del asma; aunque estudios recientes demuestran que el beneficio de este indicador en el manejo del asma no sería mejor que la evaluación de los síntomas o la función pulmonar en el ajuste de la terapia controladora $^{34-36}$.

La segunda limitación fue acotar la investigación sólo a adolescentes de 12 a 17 años y no incorporar a niños asmáticos de menor edad. A diferencia del cuestionario PAQLQ de Juniper, que fue validado en español para niños de 7 a 17 años, el cuestionario ACT aún no cuenta con validaciones en español para niños menores de 12 años, lo que no nos permitió incorporarlos al estudio. Creemos que este grupo de niños tiene la habilidad necesaria para realizar una adecuada espirometría y responder junto a sus cuidadores los nuevos cuestionarios de control de síntomas o versiones abreviadas de PAQLQ diseñados para su edad y validados recientemente en idioma inglés ${ }^{37-39}$.

Nuestros resultados sugieren que la encuesta de síntomas y la medición de calidad de vida son los instrumentos que presentaron mejor correlación y concordancia en el control del asma. Ambos instrumentos detectan el asma fuera de control antes que el valor de $\mathrm{VEF}_{1}$ sea inferior a $80 \%$ del predeterminado.

Pensamos que estos tres instrumentos no son excluyentes, sino complementarios. La evaluación de síntomas es útil para medir el control en la mayoría de los pacientes pediátricos con asma persistente, la calidad de vida permite además identificar a los pacientes con asma no controlada $\mathrm{y}$ alteraciones emocionales como adolescentes o pacientes con trastornos de salud mental. El $\mathrm{VEF}_{1}$ con el punto de corte actualmente recomendado pareciera no ser un instrumento adecuado para medir el control de asma en los niños asmáticos.

\section{Bibliografía}

1.- JUNIPER E, O'BYRNE P, GUYATT G, FERRIE P, KING D. Development and validation of a questionnaire to measure asthma control. Eur Respir J 1999; 14: 
902-7.

2.- EHRS P, NOKELA M, STÄLLBERG B, HJEMDAHL P, WIKSTRÖM JONSSON E. Brief Questionnaires for Patient- Reported Outcomes in Asthma*Validation and Usefulness in a Primary Care Setting. Chest 2006; 129: 925-32.

3.- JUNIPER E, GUYATT G, COX F, FERRIE P, KING D. Development and validation of the Mini Asthma Quality of Life Questionnaire. Eur Respir J 1999; 14: 32-38.

4.- JUNIPER E, O'BYRNE P, FERRIE P, KING D, ROBERTS J. Measuring Asthma Control Clinic Questionnaire or Daily Diary? Am J Respir Crit Care Med 2000; 162: 1330-4.

5.- JUNIPER E, BUIST S, COX F, FERRIE P, KING D. Validation of a Standardized Version of the Asthma Quality of Life Questionnaire Chest 1999; 115: 126570.

6.- Global Initiative for Asthma, Global Strategy for Asthma Management and Prevention. Diagnosis and classification. Chapter 2. Updated 2010. Available at: http:// www.ginasthma.org [Consultado el 23 diciembre 2010].

7.- REDDEL H, TAYLOR D, BATEMAN E, BOULET L, BOUSHEY H, BUSSE W, et al. An official American Thoracic Society/European Respiratory Society statement: asthma control and exacerbations: standardizing endpoints for clinical asthma trials and clinical practice. Am J Respir Crit Care Med 2009; 180(1): 59-99.

8.- JUNIPER E, GUYATT G, FEENY D, GRIFFITH L, FERRIE P. Minimum skills required by children to complete health-related quality of life instruments for asthma: comparison of measurement properties Eur Respir J 1997; 10: 2285-94.

9.- JUNIPER E, GUYATT G, FEENY D, FERRIE P, GRIFFITH L, TOWNSED M. Measuring quality of life in children with asthma. Qual Life Res 1996; 5: 35-46.

10.- RUTISHAUSER C, SAWYER S, BOND L, COFFEY C, BOWES G. Development and validation of the Adolescent Asthma Quality of Life Questionnaire (AAQOL). Eur Respir J 2001; 17(1): 52-8.

11.- SCHATZ M, SORKNESS C, LI J, MARCUS P, MURRAY J, NATHAN R, et al. Asthma control test: Reliability, validity, and responsiveness in patients not previously followed by asthma specialists. J Allergy Clin Immunol 2006; 117: 549-56.

12.- THOMAS M, KAYB S, PIKE J, WILLIAMS A, ROSENZWEIG J, HILLYERD E, et al. The Asthma Control Test (ACT) as a predictor of GINA guidelinedefined asthma control: analysis of a multinational cross-sectional survey. Primary Care Respiratory Journal 2009; 18(1): 41-9.

13.- VIDAL A, UBILLA C, DUFFAU D. Control de asma en adolescentes. Rev Med Chile 2008; 136: 859-66.

14.- VIDAL A, DUFFAU D, UBILLA C. Calidad de vida en el niño asmático y su cuidador. Rev Chil Enf Respir 2007; 23: 160-6.

15.- NATHAN R, SORKNESS C, KOSINSKI M, SCHAYZ
M, LI J, MARCUS P, et al. Development of the asthma control test: a survey for assessing asthma control. J Allergy Clin Immunol 2004; 113: 59-65.

16.- GlaxoSmithKline, Inc; QualityMetrics, Inc. Copyright 2005. Asthma Control Test. Available at: http://www. asthmacontrol.com/index_es.html (Consultado el 10 de agosto de 2010).

17.- Measurement of Health-Related Quality of Life \& Asthma Control. Paediatric asthma quality of life questionnaire (PAQLQ). http://www.qoltech.co.uk/paqlq. html (Consultado el 15 de junio de 2011).

18.- GUILBERT T, GARRIS C, JHINGRAN P, BONAFEDE M, TOMASZEWSKI K, BONUS T, et al. Asthma that is not well-controlled is associated with increased healthcare utilization and decreased quality of life J. Asthma 2011; 48:126-32.

19.- DEAN B, CALIMLIM B, SACCO P, AGUILAR D, MAYKUT R, TINKELMAN D. Uncontrolled asthma: assessing quality of life and productivity of children and their caregivers using a cross-sectional Internet-based survey Health and Quality of Life Outcomes 2010; 8: 96.

20.- SCHATZ M, MOSEN D, KOSINSKI M, VOLLMER W, MAGID D, O'CONNOR E, et al. The relationship between asthma-specific quality of life and asthma control. J Asthma 2007; 44(5): 391-5.

21.- OKELO S, WU A, KRISHNAN J, RAND C, SKINNER E, DIETTE G. Emotional quality-of-life and outcomes in adolescents with asthma. J Pediatr 2004; 145(4): 5239.

22.- ALVIM C, PICININ I, CAMARGOS P, COLOSIMO E, LASMAR L, IBIAPINA C, et al. Quality of life in asthmatic adolescents: an overall evaluation of disease control J Asthma 2009; 46: 186-90.

23.- NORDLUND B, KONRADSEN J, PEDROLETTI C, KULL I, HEDLIN G. The clinical benefit of evaluating health-related-quality-of-life in children with problematic severe asthma. Acta Paediatrica 2011; 100: 1454-60.

24.- KO F, LEUNG T, HUI D, CHU H, WONG G, WONG E, et al. Asthma Control Test correlates well with the treatment decisions made by asthma specialists. Respirology 2009; 14: 559-66.

25.- CHAN M, SITARAMAN S, DOSANJH A. Asthma control test and peak expiratory flow rate: independent pediatric asthma management tools. J Asthma 2009; 46: 1042-4.

26.- BACHARIER L, STRUNK R, MAUGER D, WHITE D, LEMANSKE R, SORKNESS C. Classifying Asthma Severity in Children: Mismatch Between Symptoms, Medication Use, and Lung Function. Am J Respir Crit Care Med 2004; 170: 426-32.

27.- BUSH A, FLEMING L. Phenotypes of refractory/severe asthma. Paediatric Respiratory Reviews 2011; 12: 17781

28.- BUSH A, SAGLANI S. Management of severe asthma 
in children. Lancet 2010; 376: 814-25.

29.- FUHLBRIGGE A, WEISS S, KUNTZ K, PALTIEL D. Forced Expiratory Volume in 1 Second Percentage Improves the Classification of Severity Among Children With Asthma. Pediatrics 2006; 118: 347-55.

30.- SPAHN J, CHERNIACK R, PAULL K, GELFAND E. Is Forced Expiratory Volume in One Second the Best Measure of Severity in Childhood Asthma? Am J Respir Crit Care Med 2004; 169: 784-6.

31.- LEVY J, WELKER-HOOD L, CLOUGHERTY J, DODSON R, STEINBACH S, HYNES H. Lung function, asthma symptoms, and quality of life for children in public housing in Boston: a case-series analysis. Environ Health 2004; 3: 13.

32.- LEBRERO A. Utilization and clinical validation of the Spanish version of the Pediatric Asthma Quality of Life Questionnaire (PAQLQ) and the Diary for Caregivers of Asthmatic Children (DCA). VALAIR study. Allergol Immunopathol (Madr). 2000; 28: 175-83.

33.- American Thoracic Society Documents ATS/ERS Recommendations for Standardized Procedures for the Online and Offline Measurement of Exhaled Lower Respiratory Nitric Oxide and Nasal Nitric Oxide, 2005 Am J Respir Crit Care Med 2005; 171: 912-30.
34.- SZEFLER S, MITCHELL H, SORKNESS C, GERGEN P, O'CONNOR G, MORGAN W, et al. Management of asthma based on exhaled nitric oxide in addition to guideline-based treatment for inner-city adolescents and young adults: a randomised controlled trial. Lancet 2008; 372: 1065-72.

35.- PETSKY H, CATES C, LI A, KYNASTON J, TURNER C, CHANG A. Tailored interventions based on exhaled nitric oxide versus clinical symptoms for asthma in children and adults. Cochrane Database Syst Rev 2009; 7: CD006340.

36.- LIU A, ZEIGER R, SORKNESS C, MAHR T, OSTROM N, BURGESS S, et al. Development and crosssectional validation of the Childhood Asthma Control Test J Allergy Clin Immunol 2007; 119: 817-25.

37.- JUNIPER E, GRUFFYDD-JONES K, WARD S, SVENSSON K. Asthma Control Questionnaire in children: validation, measurement properties, interpretation Eur Respir J 2010; 36: 1410-6.

38.- MINARD J, THOMAS N, OLAJOS-CLOW J, JUNIPER E, JIANG X, JENKINS B, et al. Lougheed Validation Of An Electronic Version Of The Mini Pediatric Asthma Quality Of Life Questionnaire (mini Paqlq) Am J Respir Crit Care Med 2011; 183:A1433.

Correspondencia a:

Dr. Alberto E. Vidal G.

Unidad de Enfermedades Respiratorias,

Hospital de Niños Roberto del Río, Santiago, Chile.

E-mail: aevgmd@yahoo.es 